\title{
Consensus Statement on Analgo-sedation in Neurocritical Care and Review of Literature
}

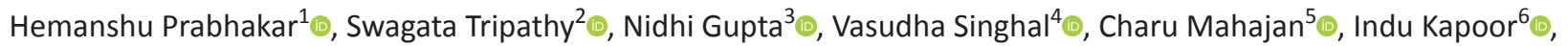 \\ Jaya Wanchoo ${ }^{7}$, Mani Kalaivani ${ }^{8}$ (B)
}

\begin{abstract}
Aim and objective: Our main objective in developing this consensus is to bring together a set of most agreed-upon statements from a panel of global experts that would act as a guide for clinicians working in neurocritical care units (NCCUs).

Background: Given the physiological benefits of analgo-sedation in the NCCU, there is little information on their tailoring in the NCCU. This lack of evidence and guidelines on the use of sedation and analgesia in patients with neurological injury leads to a variation in clinical care based on patient requirements and institutional protocols.

Review results: Thirty-nine international experts agreed to be a member of this consensus panel. A Delphi method based on a Web-based questionnaire developed with Google Forms on a secure institute server was used to seek opinions of experts. Questions were related to sedation and analgesia in the neurocritical care unit. A predefined threshold of agreement was established as $70 \%$ to support any recommendation, strong, moderate, or weak. No recommendations were made below this threshold. Responses were collected from all the experts, summated, and expressed as percentage (\%). After three rounds, consensus could be reached for 6 statements related to analgesia and 5 statements related to sedation. Consensus could not be reached for 10 statements related to analgesia and 5 statements related to sedation.

Conclusion: This global consensus statement may help in guiding practitioners in clinical decision-making regarding analgo-sedation in the NCCUs, thereby helping in improving patient recovery profiles.

Clinical significance: In the lack of high-level evidence, the recommendations may be seen as the current best clinical practice.

Keyword: Analgesia, Analgo-sedation, Consensus statement, Neurocritical care unit, Sedation.

Indian Journal of Critical Care Medicine (2021): 10.5005/jp-journals-10071-23712
\end{abstract}

\section{BACKGROUND}

The brain is often affected in critically ill patients, irrespective of the pathophysiology of the primary insult. Intensivists in the neurocritical care unit (NCCU) use sedation and analgesia more as primary treatment than as adjuvants. 'Sedation is used in the NCCU to decrease cerebral metabolic rate, anxiety, and ventilator asynchrony. It is also indicated in intracranial pressure (ICP) reduction and seizure control protocols. ${ }^{2}$ Pain is commonplace in the NCCU as a result of surgery, underlying disease, prolonged immobilization, or procedures such as endotracheal suctioning and position changing. ${ }^{3}$ Critically ill patients may experience more pain than healthy people due to hypernociception. Uncontrolled pain may increase agitation, have a deleterious effect on the ICP, and result in delirium and post-traumatic stress disorder. ${ }^{4}$

In recent times, there has been a call to tailor the use of sedatives and analgesics in general critical care patients. For example, in an attempt to decrease days on mechanical ventilation, hasten weaning, decrease delirium, and acquired ICU weakness, there has been a move toward avoiding benzodiazepines and preferring light over deep sedation. ${ }^{5}$ Given the physiological benefits of analgosedation in the NCCU, there is little information on their tailoring in the NCCU. ${ }^{6}$ This lack of evidence and guidelines on the use of sedation and analgesia in patients with neurological injury leads to a variation in clinical care based on patient requirements and institutional protocols. ${ }^{1}$ This, in turn, may affect patient outcomes such as neurocognitive recovery, delirium, and mortality. ${ }^{7,8}$ Our

\footnotetext{
1,5,6 Department of Neuroanaesthesiology and Critical Care, All India Institute of Medical Sciences, New Delhi, India

${ }^{2}$ Department of Anaesthesia, All India Institute of Medical Sciences, Bhubaneswar, Odisha, India

${ }^{3}$ Department of Neuroanesthesia, Indraprastha Apollo Hospital, New Delhi, India

4,7Department of Neuroanaesthesiology and Critical Care, Medanta: The Medicity, Gurugram, Haryana, India

${ }^{8}$ Department of Biostatistics, All India Institute of Medical Sciences, New Delhi, India
}

Corresponding Author: Hemanshu Prabhakar, Department of Neuroanaesthesiology and Critical Care, All India Institute of Medical Sciences, New Delhi, India, Phone: +91 9818727319, e-mail: prabhakaraiims@yahoo.co.in.

How to cite this article: Prabhakar H, Tripathy S, Gupta N, Singhal V, Mahajan C, Kapoor I, et al. Consensus Statement on Analgo-sedation in Neurocritical Care and Review of Literature. Indian J Crit Care Med 2021;25(2):126-133.

Source of support: Nil

Conflict of interest: None

objective in developing this consensus was to bring together a set of most-agreed-upon statements from a panel of global experts that would act as a guide for clinicians working in NCCUs. In the lack of high-level evidence, the recommendations may be seen as the current best clinical practice. 


\section{Review Results}

A Steering Committee (SC) was formed, which consisted of physician experts in the field of neurocritical care and a biostatistician. The SC prepared a questionnaire, which was then shared with a panel of renowned experts with clinical experience, from 17 countries across the globe. Experts were selected on the basis of their existing or past experience in neurocritical care. Members of the SC coordinated with these experts. The SC prepared the questionnaire to address various issues related to analgesia and sedation in neurocritical care, in terms of the need, type of drugs, timing of administration, and other practical issues. The first preliminary round was aimed at evaluating the completeness of the questionnaire and its accuracy. Questions were based on a 10-point scale ranging from totally disagree to totally agree. Experts were also encouraged to give suggestions and criticisms. The final questionnaire was developed from the results of the preliminary round. A Delphi method based on a Web-based questionnaire developed with Google Forms on a secure institute server by the authors was used to seek opinions of experts, to reduce the heterogeneity of different points of view to reach to the highest possible degree of convergence.

After the development of the questionnaire, we performed subsequent rounds to categorize recommendations into strong, moderate, and weak. After each round of questions, we analyzed the answers. For statements where consensus could be achieved, we removed them from subsequent rounds. In the subsequent rounds, questions were reframed or modified based on suggestions from the experts. If required, individual members were contacted by e-mail to confirm a correct understanding of the question and asked whether they wanted to modify or retain their answers. Members were allowed to reconsider their answers and make changes if they felt. The intention was to reach a consensus. The analysis was performed by non-voting members of the SC. We provided feedbacks to experts without disclosing the identity of members. A majority (more than $70 \%$ votes) went toward generating a recommendation (strong, moderate, or weak), which led to the formulation of the consensus statements. It was at the discretion of the expert to give a strong, moderate, or weak recommendation for these statements, based on their personal views and clinical experience. Responses were collected from all the experts and results summated.

The activity was endorsed by the Society of Neurocritical Care (SNCC), India. Thirty-nine international experts agreed to be a member of this consensus panel. The steps involved in the process of preparation of the consensus statement are shown in Flowchart 1.

In the first stage, the Steering Committee prepared 24 general statements pertinent to analgesia and 15 statements related to sedation in neurocritical care units. Once the questionnaire was finalized, the preliminary round was conducted and questions mailed to all the experts. The experts were asked to categorize their opinion on a scale of 1-10 if they totally disagreed or totally agreed with the statement. Experts were also given the liberty to reject any statement which they felt was not relevant. Based on their feedback and comments, we modified some of the statements and prepared the final set of statements. In the first round, 23 statements were sent to the experts, and a consensus was reached for 5 . In the second round, after making changes suggested by experts, a set of 22 questions were sent; consensus was reached for six statements, but no recommendations could be made for 15 statements. The statements and their recommendations/no recommendations are tabulated (Tables 1 and 2).

\section{Discussion}

Sedation and analgesia or analgo-sedation may be an integral component of intensive care of critically ill, mechanically ventilated patients for reduction of anxiety, pain, discomfort, ventilator intolerance, and patient-ventilator dyssynchrony. ${ }^{2}$ In addition, analgo-sedation in the neurocritically ill may also serve a "neurospecific" quintessential role in medical control of ICP, therapeutic temperature management for post-cardiac arrest coma and neurogenic fever, paroxysmal sympathetic activity, and seizure management. ${ }^{9}$ Analgo-sedation is, however, not without risk, particularly in the neurointensive care. Existing literature in medical and surgical intensive care units demonstrates that sedative medications can be associated with significant over-sedation and delirium, leading to prolonged stay in ICU and increased morbidity and mortality. ${ }^{10}$ This has to be balanced against the risk

Flowchart 1: Summary of the steps of formulating the consensus statement

Preliminary round: The questionnaire prepared by the Steering Committee was submitted to the experts for an evaluation of the content. Suggestions were invited, and framing of sentences, if required, was permitted.

Responses were noted and suggestions incorporated in the questionnaire, wherever feasible.

First round: Final questionnaire comprising of 'statements' was sent to the experts. They were asked to give their recommendations for each statement, as Strong, Moderate, or Weak. Experts were allowed to give their suggestions.

The results were analyzed. Individual experts were contacted if there was inconsistency in answers. Any alteration in response was allowed and 'statements' reframed.

Second round: 'Statements' were resubmitted and final opinion taken from the experts on each of the 'statement,' as 'Yes' or 'No.' Results were analyzed, and the final degree of recommendation given according to the predefined threshold.

Preparation of final document; shared with experts for approval. 
Table 1: Strong recommendations from the expert panel for consensus statement on analgo-sedation in neurocritical care (\% response)

\section{Analgesia}

1. Along with the pain scales, vital parameters (heart rate, blood pressure, respiratory rate) should be used for assessment of pain in NCC units [strong recommendation-78.9\%].

2. All neurological and neurosurgical patients should receive analgesics prior to sedatives [strong recommendation-71.1\%].

3. In mechanically ventilated patients, infusions can be the preferred mode of administration of analgesics in NCC units [strong recommendation-73.7\%].

4. Fentanyl and acetaminophen should be the preferred analgesics in NCC units [strong recommendation-76.3\%].

5. At least one of the following scales-Behavioral Pain Scale, or Critical Care Pain Observation Tool—should be used for quantification of pain in the NCC unit [strong recommendation-76.3\%].

\section{Sedation}

1. Emphasis should also be given on non-pharmacological methods of pain management (such as proper positioning of patients and nursing care) [strong recommendation-81.6\%].

2. Propofol and dexmedetomidine can be used as primary sedatives in NCC units [strong recommendation-84.2\%].

3. Richmond Agitation-Sedation Scale, Ramsay Sedation Scale, or Riker Sedation Agitation Scale should be used to monitor the level of sedation in NCC units [strong recommendation-76.3\%].

4. In general, a protocol for holding sedation every morning should be followed unless indicated otherwise [strong recommendation-81.6\%].

5. A deep level of sedation should be maintained in patients with conditions such as refractory status epilepticus, paroxysmal sympathetic activity, targeted temperature management, or refractory intracranial hypertension in NCC units [strong recommendation-78.9\%].

6. In patients with ICP catheter in situ (and normal ICP values), sedation interruptions should be given after the first few days [strong recommendation-78.9\%].

of interrupting sedatives and analgesics early, which might lead to agitation, anxiety, patient injury, device removal, and intracranial hypertension in patients with reduced brain compliance.

Despite the potential utility of tailoring analgo-sedation in the critically ill, there is a paucity of evidence for optimal sedation and analgesic management in neurologically injured patients receiving intensive care. ${ }^{6}$ This lack of evidence could be explained by either exclusion or underrepresentation of neurocritically ill patients in comparison with "general ICU patients" in most studies, on ethical grounds. ${ }^{11,12}$

Furthermore, conducting a randomized controlled trial in the NCCU presents particular challenges, considering the heterogeneous mixture of patients with different neuropathophysiology (including severe traumatic brain injury, poor-grade subarachnoid hemorrhage, severe ischemic/hemorrhagic stroke, comatose cardiac arrest, and status epilepticus), different sedation goals, and simultaneous use of several pharmacologic agents, leading to a confounding result.

Assessment and monitoring for pain and sedation in neurointensive care setups are limited by the primary pathology that may alter the level of consciousness or cause language deficits.
Table 2: No recommendations from the expert panel for statements (\% response)

\section{Analgesia}

1. In general, pain experienced by patients in NCC units is of moderate to severe intensity [strong $-52.6 \%$; moderate-47.4\%].

2. In non-ventilated patients, boluses can be the preferred mode of administration of analgesics in NCC units [strong- $52.6 \%$; moderate-47.4\%].

3. Morphine cannot be used in NCC units unless indicated otherwise [strong-15.8\%; moderate-34.2\%; weak-50\%].

4. Remifentanil can be used in NCC units [strong-28.9\%; moderate-52.6\%; weak-18.4\%].

5. NSAIDs can be used in NCC units [strong-34.2\%; moderate-42.1\%; weak-23.7\%].

6. All patients undergoing craniotomies should be given scalp blocks for analgesia postoperatively [strong-52.6\%; moderate-47.4\%].

7. Epidural analgesia can be provided in postoperative spine surgeries [moderate-52.6\%; weak-47.4\%].

8. Ketamine can be used as an adjuvant with analgesics in NCC units [strong-21.1\%; moderate-44.7\%; weak-34.2\%].

9. Dexmedetomidine can be used as an adjuvant with analgesics in NCC units [strong - 52.6\%; moderate-34.2\%; weak-13.2\%].

10. Lidocaine/lignocaine and magnesium can be used as adjuvants with analgesics in NCC units [moderate-55.3\%; weak-44.7\%].

\section{Sedation}

1. Midazolam should not be the primary agent of choice for sedation in NCC units [strong-55.3\%; moderate-44.7\%].

2. Opioids can be used as an adjunct for sedation in NCC units [strong-57.9\%; moderate-42.1\%].

3. Ketamine can be used as an adjuvant for sedation [moderate-34.2\%; weak-65.8\%].

4. In addition to the subjective clinical assessment for sedation, patients can be monitored with EEG-based monitors [strong-55.3\%; moderate-44.7\%].

5. A light level of sedation can be maintained in patients in NCC units [strong $-55.3 \%$; moderate-44.7\%].

At the same time, sedation choices may confound accurate serial neurological examination, the cornerstone of clinical assessment. Therapeutic and procedural decision-making is often contingent upon an accurate neurological assessment, which is obscured by the use of sedatives. Acute changes in brain physiology become difficult to detect, and the accuracy of neuroprognostication is decreased. Oddo et al. suggest a practical approach for the use of sedation and analgesia in the neuro-ICU, with specific attention on how to best initiate, titrate, and stop sedation, according to the severity of acute brain injury. ${ }^{9}$ In 2017 , the Neurocritical Care Society (NCS) initiated a series of protocols, termed the Emergency Neurological Life Support (ENLS), and reviewed the management of pain, agitation, and delirium in the neurocritically ill. ${ }^{13}$ However, in the absence of high-quality studies providing level-1 evidence, most of the clinical practice is based on personal and institutional choices, with no clear-cut recommendations.

\section{Analgesia}

Adequate pain control is an essential component of holistic care of critically ill patients, needless to say neurocritically ill. Severe pain 
may cause hemodynamic disturbances (tachycardia, bradycardia, hypertension, hypotension, bradypnea, and desaturation), critically jeopardizing cerebral perfusion and negatively affecting patient status (e.g., cardiac instability, respiratory compromise, ventilator distress, and immunosuppression). ${ }^{5,14}$

Evaluating pain is an integral component of initial assessment of critically ill patients, pain being the fifth vital monitor to be assessed. However, assessing the requirement and adequacy of analgesia presents special challenges in the neurocritically ill patients. Patient's self-reported pain level (Numeric Rating Scale, administered either verbally or visually) has long been considered the "gold standard" for pain evaluation in patients who can communicate reliably. ${ }^{15}$ However, it is impractical in NCCU because of the patient's impaired ability to communicate (aphasia, dysphasia), altered mental status/impaired consciousness, use of sedation, and/or mechanical ventilation. Providing adequate analgesia in the NCCU setting necessitates a delicate balance between providing painlessness and avoiding excessive sedation by carefully titrated analgesic dosing to the desired drug's effect. ${ }^{16}$

The recent clinical practice guidelines recommend using behavioral pain assessment tools such as Behavioral Pain Scale in intubated (BPS) and non-intubated (BPS-NI) patients, and the Critical Care Pain Observation Tool (CPOT) among critically ill adults, unable to self-report pain and in whom behaviors are observable. ${ }^{5,17}$ Both CPOT and the BPS have demonstrated the greatest validity and reliability for monitoring pain across large samples of general ICU patients including medical, surgical, and trauma ICUs. ${ }^{50-54}$ However, uncertainties remain about the optimal performance of these scales in brain-injured patients as critically ill traumatic brain injury (TBI) patients could exhibit atypical behaviors when exposed to nociceptive procedures. As such, the use of the currently recommended pain behaviors as part of the standardized scales may not be optimal. ${ }^{18}$ Nonetheless, multiple observational studies have revealed that using BPS, BPS-NI, and CPOT is feasible and reliable for assessing the analgesic needs of this vulnerable group in the brain-injured population. ${ }^{19-23}$ Yu et al. performed the first prospective, observational multicentered trial to assess the feasibility of systematic evaluation of pain, sedation, and delirium features in the neurologically critically ill patient, using scales validated for general ICU-intubated patients. The authors found that pain and sedation can be systematically assessed in the neurologically critically ill; the majority can also be screened for delirium features with excellent inter-rater reliability. Pain was always assessable with excellent inter-rater reliability (Numeric Rating Scale intraclass correlation coefficient, 0.92; and Behavioral Pain Scale intraclass correlation coefficient, 0.83). ${ }^{19}$

Our Consensus Committee recommends using at least one of the following scales-Behavioral Pain Scale, or the Critical Care Pain Observation Tool-for quantification of pain in the neurocritical care (NCC) unit. In addition, vital parameters (heart rate, blood pressure, respiratory rate) should also be used for assessment of pain along with the pain scales in NCC units, as in most circumstances the determination of the adequacy of analgesia for these patients still relies upon the observation of indirect signs of pain, for example, tachycardia, systemic hypertension, and elevation in ICP during painful interventions.

Though no strong consensus could be achieved regarding the intensity of pain experienced by patients in NCC units, majority considered it to be of moderate to severe in intensity. Many neurocritically ill patients, however, require only analgesia and are not candidates for sedation, unless indicated for pharmacological management of raised ICP or ongoing seizures (discussed later). Furthermore, sedation without pain control may be an important cause of agitation or delirium in critically ill. As most sedatives do not provide analgesia, the recently advocated practice of analgosedation recommends analgesia - the first technique in critically ill and mechanically ventilated populations. ${ }^{11,24,25}$ Henceforth, our Consensus Committee strongly recommends that all neurological and neurosurgical patients should receive analgesics prior to sedatives.

Continuous infusion of sedative and opioid agents has generally been considered to protect the severely injured brain in the acute phase, i.e., first 24-48 hours, as bolus dosing of analgesic agents may cause hypotension, particularly in volume-depleted patients, thereby severely compromising cerebral perfusion pressure. ${ }^{9}$ Furthermore, infusion of short-acting analgesics allows for the interruption and neurological assessment at intervals. Hence, we strongly recommend drug infusions as the preferred mode of administering analgesics in mechanically ventilated NCC patients.

Considering the choice of analgesic in the neurocritically ill, no particular pharmacological agent or class of drug has been shown to provide superior analgesic effectiveness. ${ }^{6}$ Nonetheless, on the basis of available evidence, acetaminophen and opioids have been considered as the most commonly used analgesics in NCC patients. ${ }^{27,28}$ An international survey and practice audit of six NCCUs with a small study sample of 173 patients showed acetaminophen/ paracetamol as the most common first-line analgesic, used in $49.1 \%$ of neurocritically ill patients, followed by opiates as the "second line" in $31.5 \%$ of patients, with gabapentin as a contextual third choice. ${ }^{27}$ Overall, opioids were found to be prescribed in $97 \%$ of NCCU patients. Preferred opiates were fentanyl (55.6\%), morphine (38.8\%), and hydromorphone (27.1\%), while opiate and precursor choices were sufentanil (3.0\%), remifentanil (4.5\%), oxycodone (14.6\%), and codeine (8.3\%). Acetaminophen/paracetamol doses were most often reported as $1 \mathrm{~g}$ orally every 4-6 hours (68.6\%). NSAIDs have been reported as analgesics of choice in less than $40 \%$ of the neurocritically ill patients and never chosen as a first-line drug, even in patients with postoperative pain. Short-acting opiates like remifentanil may be of greater value during the spontaneous awakening and breathing trials or the neurological wake-up tests in brain-injured patients. ${ }^{25}$

Echoing the results of all the pertinent literature on analgesia use, our Consensus Committee advocates fentanyl and acetaminophen as the preferred analgesics in NCC units. However, no consensus could be reached regarding the use of other analgesics such as morphine, remifentanil, and NSAIDs. A relevant reason for this might be the non-availability of certain opioids, like remifentanil, in the middle- and low-income group countries. Similarly, no strong recommendation could be made regarding the use of ketamine, dexmedetomidine, lidocaine/lignocaine, and magnesium as adjuvants with analgesics in NCC units. For analgesia in postoperative neurosurgical cases, no strong recommendation could be provided for scalp blocks in patients undergoing craniotomies, and epidural analgesia for patients undergoing spine surgeries. However, there is a strong recommendation for encouraging non-pharmacological means of analgesia in the form of proper positioning of patients and nursing care. Good nursing care practices like creating a peaceful environment for the patient, removing unnecessary tension from areas that are under pressure, correct method of bandaging, or an appropriate position of the patient's body go a long way in reducing the need for sedatives and analgesics in the ICU, and ensuring patient comfort. Quality 
education of nurses in this regard can play a crucial role in adopting appropriate sedation protocols.

\section{Sedation}

Given the lack of large trials which have evaluated clinically relevant endpoints, sedative selection depends on the effect of each agent on cerebral and systemic hemodynamics. This Consensus Committee strongly recommends propofol and dexmedetomidine to be used as primary sedatives in NCC units. The recent clinical practice guidelines for the management of sedation in critically ill adults also suggest using either propofol or dexmedetomidine over benzodiazepines for sedation in critically ill, mechanically ventilated adults, though the quality of evidence is still low. ${ }^{5}$ Nonetheless, both drugs offer a moderate benefit over commonly used benzodiazepine, midazolam, in terms of reduced time to achieve light sedation and extubation of critically ill adults.

Propofol is currently the most commonly used sedative agent in many NCCUs for the management of brain-injured patients, considering its favorable cerebral physiological effects (preserves CBF/CMRO2 coupling, cerebrovascular reactivity, and brain oxygenation at doses $<4 \mathrm{mg} / \mathrm{kg} / \mathrm{h}$ ) and excellent control of ICP and seizures. ${ }^{9,28}$ Standard or high-dose propofol infusion $(2 \mathrm{mg} /$ $\mathrm{kg}$ induction bolus followed by $150-200 \mu \mathrm{g} / \mathrm{kg} / \mathrm{min}$ infusion) can induce EEG burst suppression and has been recommended for the control of status epilepticus. ${ }^{29,30}$ Compared to midazolam, propofol sedation in neurocritically ill has been found to facilitate the use of spontaneous awakening trials due to its shorter half-life and thus allows for early weaning from mechanical ventilation. ${ }^{31}$ However, the use of propofol in NCCU has received intense scrutiny for its side effects, mainly hypotension and propofol infusion syndrome (PRIS). ${ }^{32}$

Recently released fourth edition guidelines for the management of severe traumatic brain injury $(\mathrm{TBI})^{33}$ reviewed the existing evidence for the safety of propofol in patients with severe TBI. It advocates for "extreme caution" when using high-dose infusions or exceeding a total usage of 48 hours, to prevent its accumulation, prolongation in half-life, and PRIS. A study, comparing propofol to dexmedetomidine sedation in the neurocritically ill, found high (30\%) incidences of hypotension in both groups. ${ }^{34}$ Caution must be utilized with these medications when concerns for brain ischemia are present.

Dexmedetomidine is a selective a2-agonist with sedative, anxiolytic, and analgesic properties. Over years, it has gained popularity in NCC units owing to its favorable drug dynamics, primarily short half-life, hemodynamic stability, minimal respiratory depressive effects, easy reversibility, proposed effectiveness with regard to patient cognition, cooperation, and facilitation of neurological examination, reduced need for adjunctive sedative and analgesic agents, lower incidence of delirium (compared to benzodiazepine, midazolam, and lorazepam), and enabling early weaning from mechanical ventilation. ${ }^{9,35-38}$ Despite a favorable clinical profile, no recommendation had earlier been made with regard to its use in neurocritically ill patients, as the evidence for its safety, mainly as a consequence of its hemodynamic effects, was lacking. ${ }^{33}$ Nevertheless, recent literature provides abundant data suggesting no significant untoward effects of dexmedetomidine on cerebral or systemic hemodynamics, and neurological functioning, when used to target light sedation in this precarious patient population. ${ }^{39-43}$

Dexmedetomidine and propofol have been proved equally effective at maintaining sedation, with no significant difference in systemic or cerebral parameters in patients with acute brain injury.

Though midazolam infusion has been found equally effective to propofol in maintaining cerebral hemodynamics, the increasingly higher doses required for such use may lead to drug bioaccumulation and an increased risk of delirium, prolonged duration of coma, mechanical ventilation, and ICU length of stay. ${ }^{44,45}$ No strong recommendation could be made regarding the use of midazolam in neurocritically ill by our Consensus Statement Group. Likewise, only $57.9 \%$ of respondents strongly believed that opioids can be used as an adjunct for sedation in NCC units.

Ketamine is a newer adjunct in neuroanesthetic armamentarium and is primarily used for managing refractory and super-refractory status epilepticus. ${ }^{45}$ Despite its proposed beneficial effects such as maintaining respiratory drive and systemic hemodynamics, and anti-excitatory neuroprotective effects, ${ }^{9}$ it is still not recommended for sedation by majority of neurointensivists in our Consensus Group, possibly out of concerns of raised intracranial pressure and lack of safety data in the neurocritically ill.

For monitoring the level of sedation, conventional validated sedation scoring tools for critical care, such as the Richmond Agitation-Sedation Scale (RASS), Ramsay Sedation Scale, or Riker Sedation Agitation Scale, should be used to monitor the level of sedation in NCC units. These scales, originally developed for general critically ill patients, have been well validated with excellent interrater reliability in neurologically injured patients as well. ${ }^{19,46,47}$

In addition to the subjective clinical assessment for sedation, as per the Consensus Committee, patients can also be monitored with EEG-based monitors, though no strong recommendation could be made supporting this statement. However, studies in neurologically injured patients support the utility of EEG-based tools in patients receiving deep levels of sedation and/or neuromuscular blocking agents, with BIS levels significantly correlating with routine sedation scale monitoring. ${ }^{47-49}$ Nevertheless, caution should be exercised regarding the interpretation of BIS in TBI, as brain injury itself influences BIS signals in addition to the confounding effects of shivering.

In general, recent guidelines on the management of sedation in critically ill do not provide any strong consensus on the level of sedation to be maintained in critically ill, with a conditional recommendation to maintain light sedation to achieve better clinical outcomes. ${ }^{5}$ Evidently, there is a dearth of literature on the relationship between the depth of sedation and neurocognitive function, clinical outcome, and all-cause mortality in NCCU patients. In our Consensus Group, a light level of sedation is preferred by only a half of respondents, whereas a deeper level of sedation is strongly recommended in patients with critical neural injury, such as those with refractory status epilepticus, paroxysmal sympathetic activity, refractory intracranial hypertension, or undergoing targeted temperature management for hyperthermia. Likewise, these patients should not be subjected to the spontaneous awakening trials during the intense therapy duration. In patients undergoing intracranial pressure (ICP) monitoring for resistant intracranial hypertension, it is strongly recommended that an awakening trial or sedation interruption should be attempted only after the first few days of therapy (with ICP in near-normal values), to prevent an unwarranted increase in ICP.

Other than these exceptions, it is again strongly recommended that a sedation interruption or lightening of sedation protocol should be followed every morning to facilitate early weaning from mechanical ventilation. To date, numerous controversies 
remain with regard to interruption of sedation in TBI patients where continuous sedation is part of the treatment strategy. ${ }^{50}$ In the only randomized controlled trial addressing a daily interruption of continuous sedation (DIS) protocol in a small subgroup of TBI patients, significantly decreased days on ventilation or length of stay in the NCC were not observed. ${ }^{51}$ The proponents of sedation interruption in neurocritically ill argue that even in the presence of advanced neuromonitoring tools, this is the only reliable means to timely detect clinically important neurological improvement or deterioration. ${ }^{52}$ It may further aid in clinical decision-making regarding the urgent need for radiological confirmation of the cause of neurological deterioration, need for surgical decompression, or change in ventilatory parameters. Nonetheless, titration and withdrawal of sedation in the NCCU setting have to be balanced between the risk of sedation interruption leading to significant systemic stress response and increase in ICP, against the perceived benefits of enhanced neurological function and reduced complications. ${ }^{53-55}$ The need for physical restraints for patient and device safety during periods of sedation interruption and the role of bedside nurses as the main decision-makers in restraint management need to be emphasized.

Skoglund et al. evaluated the effects of interruption of sedation in patients with TBI using advanced neuromonitoring tools (ICP monitoring, cerebral microdialysis, brain tissue oxygenation $\left(\mathrm{PbtO}_{2}\right)$, jugular venous oxygen saturation, and/or arterial-venous difference for glucose, lactate, and oxygen) and found that despite a significant increase in ICP and CPP, sedation interruption did not negatively affect brain biochemistry or cerebral oxygenation. ${ }^{56}$ Keeping in my mind the pros and cons of sedation interruption in the neurocritically ill, it should only be attempted in patients showing stable ICP, CPP, and $\mathrm{PbtO}_{2}$ (if available) values during continuous sedation and should be withheld in patients with unstable ICP and/or CPP levels, hyperthermia, status epilepticus, and/ or barbiturate treatment. ${ }^{9,50}$ Such patients should ideally undergo graded sedation withdrawal with standard ICP or $\mathrm{PbtO}_{2}$ targets as specified by the treating neurointensivists. At this stage, it might be imperative to say that individualizing analgo-sedation practices for a patient-targeted approach to control delirium in the NCCU remains the way forward.

One of the limitations of this consensus statement might be the expert panel exclusively of physicians. With the emerging role of clinical pharmacists, who contribute to the analgo-sedation protocols and practices in the NCCUs worldwide, inclusion of this specialty would have probably given some more insights. In our questionnaire, we did not include statements pertaining to the possible role of volatile anesthetics in NCCUs, for the fact that it is still not a popular technique. However, in the present times of the coronavirus disease pandemic, one might consider the possible usage of devices such as AnaConDa ${ }^{\oplus}$. We kept a lower threshold of $70 \%$ to reach to a consensus for as many statements as possible, unlike the usual cutoff margin of $75 \%$. This was due to the fact that being a panel of global experts, with different practices in different regions, reaching to a consensus with more than $75 \%$ would have been difficult and many more statements would have remained without conclusive decision. This article is also limited by the fact that we have not included any systematic search strategy and results on analgo-sedation in neurocritical care patients. It is emphasized that at the time of formulating these consensus statements, they are not an official guideline from any society, but recommendations from a global expert panel.

\section{Conclusion}

A "bundled" approach to decrease ICU stay, among other evidencebased elements, includes an optimal choice of sedation and analgesia in the neurocritically ill patients. The active role played by the clinical pharmacists in the analgo-sedation choice and administration for NCCU patients and higher quality education of the bedside nurses on sedation and pain control, along with non-pharmacological methods, need to be highlighted. The effectiveness of using volatile anesthetic agents for "nontraditional" ICU sedation using special device, for rapid titration and faster wake-up, needs to be explored as well.

\section{Clinical Significance}

This consensus statement, prepared by the contribution of leading experts in the field of neurocritical care from across the globe, may help in guiding practitioners in clinical decision-making regarding analgo-sedation in the neuro-ICUs, thereby helping in improving patient recovery profiles.

\section{OrCID}

Hemanshu Prabhakar 이 https://orcid.org/0000-0001-7830-3296

Swagata Tripathy 10 https://orcid.org/0000-0002-5315-6477

Nidhi Gupta @ https://orcid.org/0000-0002-6058-375X

Vasudha Singhal @ https://orcid.org/0000-0002-6986-0880

Charu Mahajan — https://orcid.org/0000-0003-3712-9281

Indu Kapoor (1) https://orcid.org/0000-0002-3065-4940

Jaya Wanchoo @ ittps://orcid.org/0000-0002-9854-4916

Mani Kalaivani 아 https://orcid.org/0000-0002-6492-1808

\section{References}

1. Vanaclocha N, Chisbert V, Quilis V, Bilotta F, Badenes R. Sedation during neurocritical care. J Neuroanaesth Crit Care 2019;6:56-61. DOI: 10.1055/s-0039-1688897.

2. Chester K, Greene KN, Brophy GM. Sedation in the critical care unit. In: Prabhakar H, Ali Z. Textbook of Neuroanesthesia and Neurocritical Care, Vol II, 1st ed. Singapore: Springer; 2019. p. 299-318. DOI: 10.1007/978-981-13-3390-3_21.

3. Fischer M, Jackson M, Abd-Elsayed A. Pain in the neurocritical care unit. In: Prabhakar H, Ali Z. Textbook of Neuroanesthesia and Neurocritical Care, Vol II, 1st ed. Singapore: Springer; 2019. p. 319-331. DOI: 10.1007/978-981-13-3390-3_22.

4. Hannawi Y, Ziai WC. Analgesia, sedation, and paralysis in the neurocritical care Unit. In: Torbey MT. Neurocritical Care, 2nd ed. Cambridge: Cambridge University Press; 2019. p. 33-49. DOI: 10.1017/9781107587908.005.

5. Devlin JW, Skrobik Y, Gélinas C, Needham DM, Slooter AJC, Pandharipande PP, et al. Clinical practice guidelines for the prevention and management of pain, agitation/sedation, delirium, immobility, and sleep disruption in adult patients in the ICU. Crit Care Med 2018;46(9):e825-e873. DOI: 10.1097/CCM.0000000000003299.

6. Teitelbaum JS, Ayoub O, Skrobik Y. A critical appraisal of sedation, analgesia and delirium in neurocritical care. Can J Neurol Sci 2011;38(6):815-825. DOI: 10.1017/s0317167100012385.

7. May TL, Riker RR, Fraser GL, Hirsch KG, Agarwal S, Duarte C, et al. Variation in sedation and neuromuscular blockade regimens on outcome after cardiac arrest. Crit Care Med 2018;46(10):e975-e980. DOI: 10.1097/CCM.0000000000003301.

8. Meyfroidt G, Smith M. Focus on delirium, sedation and neuro critical care 2019: towards a more brain-friendly environment? Intensive Care Med 2019;45(9):1292-1294. DOI: 10.1007/s00134-019-05701-2.

9. Oddo M, Crippa IA, Mehta S, Menon D, Payen J-F, Taccone FS, et al. Optimizing sedation in patients with acute brain injury. Crit Care 2016;20(1):128. DOI: 10.1186/s13054-016-1294-5. 
10. Roberts DJ, Haroon B, Hall RI. Sedation for critically ill or injured adults in the intensive care unit: a shifting paradigm. Drugs. 2012;72(14):1881-1916. DOI: 10.2165/11636220-000000000-00000.

11. Barr J, Fraser GL, Puntillo K, et al. Clinical practice guidelines for the management of pain, agitation, and delirium in adult patients in the intensive care unit. Crit Care Med 2013;41(1):263-306. DOI: 10.1097/ CCM.0b013e3182783b72.

12. Skrobik $Y$, Chanques $G$. The pain, agitation, and delirium practice guidelines for adult critically ill patients: a post-publication perspective. Ann Intensive Care 2013;3(1):9. DOI: 10.1186/21105820-3-9.

13. Rajajee V, Riggs B, Seder DB. Emergency neurological life support: airway, ventilation, and sedation. Neurocrit Care 2017;27(Suppl 1):4-28. DOI: 10.1007/s12028-017-0451-2.

14. de Jong A, Molinari N, de Lattre S, et al. Decreasing severe pain and serious adverse events while moving intensive care unit patients: a prospective interventional study (the NURSE-DO project). Crit Care 2013;17(2):R74. DOI: $10.1186 / \mathrm{cc} 12683$.

15. Merskey $\mathrm{H}$, Bogduk N. Classification of chronic pain: descriptions of chronic pain syndromes and definitions of pain terms. Task Force on Taxonomy of the International Association for the Study of Pain. 2nd ed. Seattle, WA: IASP Press; 1994.

16. Devlin JW, Marquis F, Riker RR, Robbins T, Garpestad E, Fong JJ, et al. Combined didactic and scenario-based education improves the ability of intensive care unit staff to recognize delirium at the bedside. Crit Care 2008;12(1):R19. DOI: 10.1186/cc6793.

17. Herr K, Coyne PJ, Ely E, Gélinas C, Manworren RCB. Pain assessment in the patient unable to self-report: clinical practice recommendations in support of the ASPMN 2019 position statement. Pain Manag Nurs 2019;20(5):404-417. DOI: 10.1016/j.pmn.2019.07.005.

18. Arbour C, Choiniere M, Topolovec-Vranic J, Loiselle CG, Puntillo K, Gélinas $C$. Detecting pain in traumatic brain-injured patients with different levels of consciousness during common procedures in the ICU: typical or atypical behaviors? Clin J Pain 2014;30(11):960-969. DOI: 10.1097/AJP.0000000000000061.

19. Yu A, Teitelbaum J, Scott J, Gesin G, Russell B, Huynh T, et al. Evaluating pain, sedation, and delirium in the neurologically critically ill-feasibility and reliability of standardized tools: a multiinstitutional study. Crit Care Med 2013;41(8):2002-2007. DOI: 10.1097/ CCM.0b013e31828e96c0.

20. Dehghani $\mathrm{H}$, Tavangar $\mathrm{H}$, Ghandehari A. Validity and reliability of behavioral pain scale in patients with low level of consciousness due to head trauma hospitalized in intensive care unit. Arch Trauma Res 2014;3(1):e18608. DOI: 10.5812/atr.18608.

21. Echegaray-Benites $C$, Kapoustina $O$, Gélinas $C$. Validation of the use of the Critical-Care Pain Observation Tool (CPOT) with brain surgery patients in the neurosurgical intensive care unit. Intensive Crit Care Nurs 2014;30(5):257-265. DOI: 10.1016/j.iccn.2014.04.002.

22. Joffe AM, McNulty B, Boitor M, Marsh R, Gélinas C. Validation of the Critical-Care Pain Observation Tool in brain-injured critically ill adults. J Crit Care 2016;36:76-80. DOI: 10.1016/j.jcrc.2016.05.011.

23. Shan K, Cao W, Yuan Y, Hao J-J, Sun X-M, He X, et al. Use of the criticalcare pain observation tool and the bispectral index for the detection of pain in brain-injured patients undergoing mechanical ventilation: a STROBE-compliant observational study. Medicine (Baltimore) 2018:97(22):e10985. DOI: 10.1097/MD.0000000000010985.

24. Devabhakthuni S, Armahizer MJ, Dasta JF, Kane-Gill SL. Analgosedation: a paradigm shift in intensive care unit sedation practice. Ann Pharmacother 2012;46(4):530-540. DOI: 10.1345/aph.

25. Karabinis A, Mandragos K, Stergiopoulos S, Komnos A, Soukup J, Speelberg B, et al. Safety and efficacy of analgesia-based sedation with remifentanil versus standard hypnotic-based regimens in intensive care unit patients with brain injuries: a randomised, controlled trial [ISRCTN50308308]. Crit Care 2004;8(4):R268-280. DOI: 10.1186/cc2896.

26. Skrobik Y, Ahern S, Leblanc M, Marquis F, Awissi DK, Kavanagh BP. Protocolized intensive care unit management of analgesia, sedation, and delirium improves analgesia and subsyndromal delirium rates. Anesth Analg 2010;111(2):451-463. DOI: 10.1213/ ANE.0b013e3181d7e1b8.

27. Zeiler FA, AISubaie F, Zeiler K, Bernard F, Skrobik Y. Analgesia in neurocritical care: an international survey and practice audit. Crit Care Med 2016;44(5):973-980. DOI: 10.1097/CCM.0000000000001602.

28. Brain Trauma Foundation; American Association of Neurological Surgeons; Congress of Neurological Surgeons; Guidelines for the management of severe traumatic brain injury. XI. Anesthetics, analgesics, and sedatives. J Neurotrauma 2007;24 Suppl 1:S71-S76. DOI: 10.1089/neu.2007.9985.

29. Brophy GM, Bell R, Claassen J, Alldredge B, Bleck TP, Glauser T, et al. Guidelines for the evaluation and management of status epilepticus. Neurocrit Care 2012;17(1):3-23. DOI: 10.1007/s12028-012-9695-z.

30. Glauser T, Shinnar S, Gloss D, Alldredge B, Arya R, Bainbridge J, et al. Evidence-based guideline: treatment of convulsive status epilepticus in children and adults: report of the guideline committee of the american epilepsy society. Epilepsy Curr 2016;16(1):48-61. DOI: 10.5698/1535-7597-16.1.48.

31. Skoglund K, Enblad P, Marklund N. Monitoring and sedation differences in the management of severe head injury and subarachnoid hemorrhage among neurocritical care centers. J Neurosci Nurs 2013;45(6):360-368. DOI: 10.1097/JNN.0b013e3182a3cf4f.

32. Hemphill S, McMenamin L, Bellamy MC, Hopkins PM. Propofol infusion syndrome: a structured literature review and analysis of published case reports. Br J Anaesth 2019;122(4):448-459. DOI: 10.1016/j.bja.2018.12.025.

33. Carney N, Totten AM, O'Reilly C, Ullman JS, Hawryluk GWJ, Bell MJ, et al. Guidelines for the management of severe traumatic brain injury, fourth edition. Neurosurgery 2017;80(1):6-15. DOI: 10.1227/ NEU. 0000000000001432 .

34. Jakob SM, Ruokonen E, Grounds RM, Sarapohja T, Garratt C, Pocock SJ, et al. Dexmedetomidine vs midazolam or propofol for sedation during prolonged mechanical ventilation: two randomized controlled trials. JAMA 2012;307(11):1151-1160. DOI: 10.1001/jama.2012.304.

35. Pandharipande PP, Pun BT, Herr DL, Maze M, Girard TD, Miller RR, et al. Effect of sedation with dexmedetomidine vs lorazepam on acute brain dysfunction in mechanically ventilated patients: the MENDS randomized controlled trial. JAMA 2007;298(22):2644-2653. DOI: 10.1001/jama.298.22.2644.

36. Hoy SM, Keating GM. Dexmedetomidine: a review of its use for sedation in mechanically ventilated patients in an intensive care setting and for procedural sedation. Drugs 2011;71(11):1481-1501. DOI: 10.2165/11207190-000000000-00000.

37. Tang JF, Chen PL, Tang EJ, May TA, Stiver SI. Dexmedetomidine controls agitation and facilitates reliable, serial neurological examinations in a non-intubated patient with traumatic brain injury. Neurocrit Care 2011;15(1):175-181. DOI: 10.1007/s12028-009-9315-8.

38. Humble SS, Wilson LD, Leath TC, Marshall MD, Sun DZ, Pandharipande $P P$, et al. ICU sedation with dexmedetomidine after severe traumatic brain injury. Brain Inj 2016;30(10):1266-1270. DOI: 10.1080/02699052.2016.1187289.

39. Tsaousi GG, Lamperti M, Bilotta F. Role of dexmedetomidine for sedation in neurocritical care patients: a qualitative systematic review and meta-analysis of current evidence. Clin Neuropharmacol 2016;39(3):144-151. DOI: 10.1097/WNF.0000000000000151.

40. Tran A, Blinder H, Hutton B, English SW. A systematic review of alpha-2 agonists for sedation in mechanically ventilated neurocritical care patients. Neurocrit Care 2018;28(1):12-25. DOI: 10.1007/s12028-0170388-5.

41. Carelli S, De Pascale G, Filetici N, Bocci MG, Maresca GM, Cutuli SL, et al. The place of dexmedetomidine light sedation in patients with acute brain injury. Crit Care 2019;23(1):340. DOI: 10.1186/s13054-0192637-9.

42. James ML, Olson DM, Graffagnino C. A pilot study of cerebral and haemodynamic physiological changes during sedation with dexmedetomidine or propofol in patients with acute brain injury. Anaesth Intensive Care 2012;40(6):949-957. DOI: 10.1177/0310057X1204000605. 
43. Erdman MJ, Doepker BA, Gerlach AT, Phillips GS, Elijovich L, Jones GM. A comparison of severe hemodynamic disturbances between dexmedetomidine and propofol for sedation in neurocritical care patients. Crit Care Med 2014;42(7):1696-1702. DOI: 10.1097/ CCM.0000000000000328.

44. Roberts DJ, Hall RI, Kramer AH, Robertson HL, Gallagher CN, Zygun DA. Sedation for critically ill adults with severe traumatic brain injury: a systematic review of randomized controlled trials. Crit Care Med 2011;39(12):2743-2751. DOI: 10.1097/CCM.0b013e318228236f.

45. Rai S, Drislane FW. Treatment of refractory and super-refractory status epilepticus. Neurotherapeutics 2018;15(3):697-712. DOI: 10.1007/ s13311-018-0640-5.

46. Riker RR, Fugate JE, Participants in the International Multi-disciplinary Consensus Conference on Multimodality Monitoring. Clinical monitoring scales in acute brain injury: assessment of coma, pain, agitation, and delirium. Neurocrit Care 2014;21 Suppl 2:S27-S37. DOI: 10.1007/s12028-014-0025-5.

47. Deogaonkar A, Gupta R, DeGeorgia M, Sabharwal V, Gopakumaran B, Schubert A, et al. Bispectral Index monitoring correlates with sedation scales in brain-injured patients. Crit Care Med 2004;32(12):2403-2406. DOI: 10.1097/01.ccm.0000147442.14921.a5.

48. Ogilvie MP, Pereira BMT, Ryan ML, Gomez-Rodriguez JC, Pierre EJ, Livingstone AS, et al. Bispectral index to monitor propofol sedation in trauma patients. J Trauma 2011;71(5):1415-1421. DOI: 10.1097/ TA.0b013e3182178b8b.

49. Olson DM, Thoyre SM, Peterson ED, Graffagnino C. A randomized evaluation of bispectral index-augmented sedation assessment in neurological patients. Neurocrit Care 2009;11(1):20-27. DOI: 10.1007/ s12028-008-9184-6.

50. Marklund $\mathrm{N}$. The neurological wake-up test-a role in neurocritical care monitoring of traumatic brain injury patients? Front Neurol 2017;8:540. DOI: 10.3389/fneur.2017.00540.

51. Anifantaki S, Prinianakis G, Vitsaksaki E, Katsouli V, Mari S, Symianakis $A$, et al. Daily interruption of sedative infusions in an adult medical-surgical intensive care unit: randomized controlled trial. J Adv Nurs 2009;65(5):1054-1560. DOI: 10.1111/j.13652648.2009.04967.x.

52. Helbok R, Badjatia N. Is daily awakening always safe in severely brain injured patients?. Neurocrit Care 2009;11(2):133-134. DOI: 10.1007/ s12028-009-9262-4.

53. Helbok R, Kurtz P, Schmidt MJ, Stuart MR, Fernandez L, Connolly SE, et al. Effects of the neurological wake-up test on clinical examination, intracranial pressure, brain metabolism and brain tissue oxygenation in severely brain-injured patients. Crit Care 2012;16(6):R226. DOI: 10.1186/cc11880.

54. Skoglund K, Enblad P, Marklund N. Effects of the neurological wake-up test on intracranial pressure and cerebral perfusion pressure in brain-injured patients. Neurocrit Care 2009;11(2):135-142. DOI: 10.1007/s12028-009-9255-3.

55. Skoglund K, Enblad P, Hillered L, Marklund N. The neurological wake-up test increases stress hormone levels in patients with severe traumatic brain injury. Crit Care Med 2012;40(1):216-222. DOI: 10.1097/ CCM.0b013e31822d7dbd.

56. Skoglund K, Hillered L, Purins K, Tsitsopoulos PP, Flygt J, Engquist H, et al. The neurological wake-up test does not alter cerebral energy metabolism and oxygenation in patients with severe traumatic brain injury. Neurocrit Care 2014;20(3):413-426. DOI: 10.1007/s12028-0139876-4.

\section{List of Collaborators / Experts}

1. Babi Marc-Alain (USA)

2. Bilotta Federico (Italy)

3. Botero Luis F (Colombia)

4. Chakraborty Indranil (USA)

5. Chan Matthew TV (Hong Kong)

6. de Riva Nicolás (Spain)

7. Flexman Alana (Canada)

8. Giraldo Mauricio (Canada)

9. Goettel Nicolai (Switzerland)

10. Hinduja Archana (USA)

11. Kawaguchi Masahiko (Japan)

12. Khanna Ashish K (USA)

13. Kirkman Matthew A (UK)

14. Koerner Ines (USA)

15. Latronico Nicola (Italy)

16. Legriel Stephane (Italy)

17. Lele Abhijit V (USA)

18. Paranhos Jorge (Brazil)

19. Petrikov Sergey (Russia)

20. Popugaev Konstantin (Russia)

21. Prisco Lara (UK)

22. Raheem M Samy Abdel (Egypt)

23. Rao Umamaheswara GS (India)

24. Rubiano Andres M (Colombia)

25. Santos Christiano (USA)

26. Shrestha Gentle (Nepal)

27. Soriano Sulpicio (USA)

28. Souter Mike (USA)

29. Steiner Luzius A (Switzerland)

30. Tavares Cristiane (Brazil)

31. Thal Serge (Germany)

32. Videtta Walter (Argentina)

33. Vitali Sally (USA) 